\title{
Imaging Changes in Very Young Children with Brain Tumors Treated with Proton Therapy and Chemotherapy
}

\author{
N.D. Sabin, T.E. Merchant, J.H. Harreld, Z. Patay, P. Klimo, Jr, I. Qaddoumi, G.T. Armstrong,
} K. Wright, J. Gray, D.J. Indelicato, and A. Gajjar

\begin{abstract}
SUMMARY: PT promises to reduce side effects in children with brain tumors by sparing normal tissue compared with 3D conformal or intensity-modulated radiation therapy. Information is lacking about the combined effects of PT and chemotherapy in young children. We describe imaging changes in 8 very young children with localized brain tumors who received PT after chemotherapy. Mostly transient signal abnormalities and enhancement in brain parenchyma were observed by serial MR imaging, which were consistent with radiation-induced effects on normal-appearing tissue. Correlation with PT planning data revealed that the areas of imaging abnormality were located within or adjacent to the volume that received the highest radiation dose. Radiologists should be aware of these findings in children who receive PT after chemotherapy. In this report, we describe the time course of these PT-related imaging findings and correlate them with treatment and clinical outcomes.
\end{abstract}

ABBREVIATIONS: $\mathrm{AT} / \mathrm{RT}=$ atypical teratoid rhabdoid tumor; $\mathrm{CGE}=$ cobalt gray equivalent; $\mathrm{CPC}=$ choroid plexus carcinoma; $\mathrm{PNET}=$ primitive neuroectodermal tumor; PT = proton therapy

$\mathbf{R}$ adiation therapy is the most effective adjuvant therapy in children with CNS tumors. Historically its use has been limited in very young children because of concern for late effects in normal tissues impacting neurologic, endocrine (growth and development), and cognitive outcomes. On the basis of the limited side effects observed in the treatment of children with conformal and intensity-modulated radiation therapy by using photons, the use of radiation therapy in the front-line management of very young children with localized brain tumors has been reintroduced during the past decade. ${ }^{1}$

PT is a method of radiation therapy that may further decrease

Received April 9, 2012; accepted after revision May 14.

From the Departments of Radiological Sciences (N.D.S., T.E.M., J.H.H., Z.P., J.G.), Surgery (P.K.), Oncology (I.Q., K.W., A.G.), and Epidemiology and Cancer Control (G.T.A.), St. Jude Children's Research Hospital, Memphis, Tennessee; Semmes-Murphey Neurologic and Spine Institute (P.K.), Memphis, Tennessee; Department of Neurosurgery (P.K.), University of Tennessee Health Science Center, Memphis,

Tennessee; Department of Radiation Oncology (D.J.I.), University of Florida College of Medicine, Gainesville, Florida; and University of Florida Proton Therapy Institute (D.J.I.), Jacksonville, Florida.

This work was supported in part by the National Cancer Institute through a Cancer Center Support grant (CA21765), the Noyes Brain Tumor Foundation, Musicians Against Childhood Cancer, and the American Lebanese Syrian Associated Charities. Previously presented in part at: Annual Meeting of the American Society of Neuroradiology, April 21-26, 2012; New York, New York.

Please address correspondence to: Noah D. Sabin, MD, JD, Division of Diagnostic Imaging, Department of Radiological Sciences, St. Jude Children's Research Hospital, 262 Danny Thomas PI (MS-220), Memphis, TN 38105; e-mail address:

noah.sabin@stjude.org

http://dx.doi.org/10.3174/ajnr.A3219

side effects because the physical properties of protons result in significant reductions in the dose to nontargeted normal tissues. ${ }^{2}$ In particular, PT appears ideally suited for the treatment of brain tumors in very young children to avoid or minimize the potential late effects associated with even low-dose radiation on normal tissue. The improved dose distribution of PT may permit intensification of chemotherapy in multitechnique regimens, previously prohibited by the synergistic effects of chemotherapy and radiation dose in nontargeted normal tissue. However, limited information is available about the potential side effects of PT in very young children, owing to the scant number of patients treated with this technique.

We designed and activated a prospective study in 2007 for very young children (3 years of age or younger) with CNS embryonal tumors, including medulloblastoma, supratentorial PNET, and AT/RT. The intermediate risk arm of the study included 4 cycles of postsurgical induction chemotherapy administered for 4 months, followed by focal irradiation and maintenance chemotherapy. The study was later amended to include other histopathologic subtypes (ependymoma and choroid plexus carcinoma), older patients with CNS embryonal tumors, and the option of PT.

Children in this series were treated with the protocol-specified treatment regimen and prospectively followed after treatment; this follow-up provided unique insight into the temporal nature and possible clinical and treatment factors that may contribute to posttreatment phenomena. Transient imaging changes were ob- 
Table 1: Clinical data for 8 patients with imaging changes after proton therapy

\begin{tabular}{cccccc}
\hline Patient & Sex Diagnosis & \multicolumn{1}{c}{$\begin{array}{c}\text { Location } \\
\text { Age at } \\
\text { Diagnosis } \\
\text { (yr) }\end{array}$} & $\begin{array}{c}\text { Age at } \\
\text { Proton } \\
\text { Therapy (yr) }\end{array}$ \\
\hline 1 & F & EP & Fourth ventricle & 1.7 & 2.2 \\
2 & M & EP & Fourth ventricle & 1.9 & 2.4 \\
3 & F & CPC & Left lateral ventricle & 1.2 & 2.6 \\
4 & F & PNET & Left parietal lobe & 2.7 & 3.2 \\
5 & F & MB & Fourth ventricle & 1 & 1.4 \\
6 & F & EP & Fourth ventricle & 3 & 3.5 \\
7 & F & AT/RT & Posterior fossa & 0.6 & 1 \\
8 & M & EP & Fourth ventricle & 2.2 & 2.6 \\
Median & & & & 1.8 & 2.5 \\
Mean & & & & 1.8 & 2.4 \\
\hline
\end{tabular}

Note:-EP indicates ependymoma; MB, medulloblastoma.

served early after PT in some patients. Because clinical and MR imaging changes after PT are not well-documented, we describe the time course of imaging changes associated with PT correlated with treatment and clinical outcomes.

\section{CASE SERIES}

Children with localized (nonmetastatic) CNS embryonal tumors (excluding desmoplastic medulloblastoma), ependymoma, and $\mathrm{CPC}$ were treated in an institutional review board-approved clinical trial of postsurgical induction chemotherapy, focal irradiation, and maintenance chemotherapy. The induction chemotherapy regimen consisted of four 28-day cycles of high-dose methotrexate, cyclophosphamide, cisplatin, and vincristine. A second surgery was performed as appropriate before radiation therapy for those with residual disease after 2-4 cycles of induction chemotherapy. All patients were to receive focal radiation to a dose of $54 \mathrm{~Gy}$ administered over 30 fractions by using a 5-mm anatomically confined clinical target volume margin surrounding the residual tumor and/or tumor bed. The planning target volume margin was a 3-mm geometric expansion of the clinical target volume. A total of 18 patients participating in the clinical trial have received PT rather than photon-based therapy to date. Postirradiation chemotherapy included oral cyclophosphamide; topotecan and erlotinib (medulloblastoma and ependymoma); or oral cyclophosphamide, topotecan, and etoposide (other diagnoses) for 6 months.

One of the 18 patients treated with PT for a transtentorial PNET developed progressive disease while receiving PT. This child did not complete treatment and died just over 2 months after therapy was stopped with no post-PT follow-up imaging. Seventeen patients completed PT as part of their treatment regimen. Eight of the 17 patients (47\%) developed changes on brain MR imaging after irradiation and are the subject of this report. Clinical and treatment data for these patients are shown in Table 1. There were few deviations from the chemotherapy regimen. One patient received an additional induction course of cyclophosphamide, carbo- platin, and etoposide; and 1 patient did not receive maintenance chemotherapy. All had follow-up MR imaging studies of the brain performed within a median of 1 week (range, 1-3 weeks) after completion of PT and at 3-month intervals as required by the protocol. Maintenance chemotherapy was initiated within 2 weeks of completing PT. MR imaging examinations consisted of pre- and postcontrast T1-weighted, precontrast T2-weighted, pre- and postcontrast FLAIR, postcontrast sagittal magnetization-prepared rapid acquisition of gradient echo, axial diffusionweighted and axial susceptibility-weighted pulse sequences. Intravenous gadopentetate dimeglumine (Magnevist; Bayer HealthCare Pharmaceuticals, Wayne, New Jersey) was given before obtaining the postcontrast images.

On follow-up MR imaging studies of these 8 children, there was evidence of radiation-related brain parenchymal changes within intracranial tissue structures that demonstrated no abnormal signal or enhancement before PT (Figs 1-3). The locations of these findings and accompanying time courses are shown in Table 2. Imaging changes included hyperintensity on T2-weighted and FLAIR images in the affected structures with corresponding low signal intensity on precontrast T1-weighted images. For 5 of the 8 patients, foci of restricted water diffusion were present in the areas of abnormality. One of the patients who had findings in posterior fossa structures also had abnormalities in the upper cervical spinal cord. Lesions in the brain stem, cerebellum, and upper cervical spinal cord enhanced; however, an area of signal abnormality in the right thalamus in 1 patient did not. Three of the children with brain stem lesions and the child with the right thalamic lesion underwent dynamic susceptibility contrast perfusion MR imaging, which provided satisfactory evaluation of the areas of signal abnormality. The regions of signal change in these 4 patients were hypoperfused.

Subsequent brain MR imaging examinations of 5 of the 6 patients with posterior fossa lesions performed 2.2-3.5 months after the appearance of the abnormalities showed a significant decrease
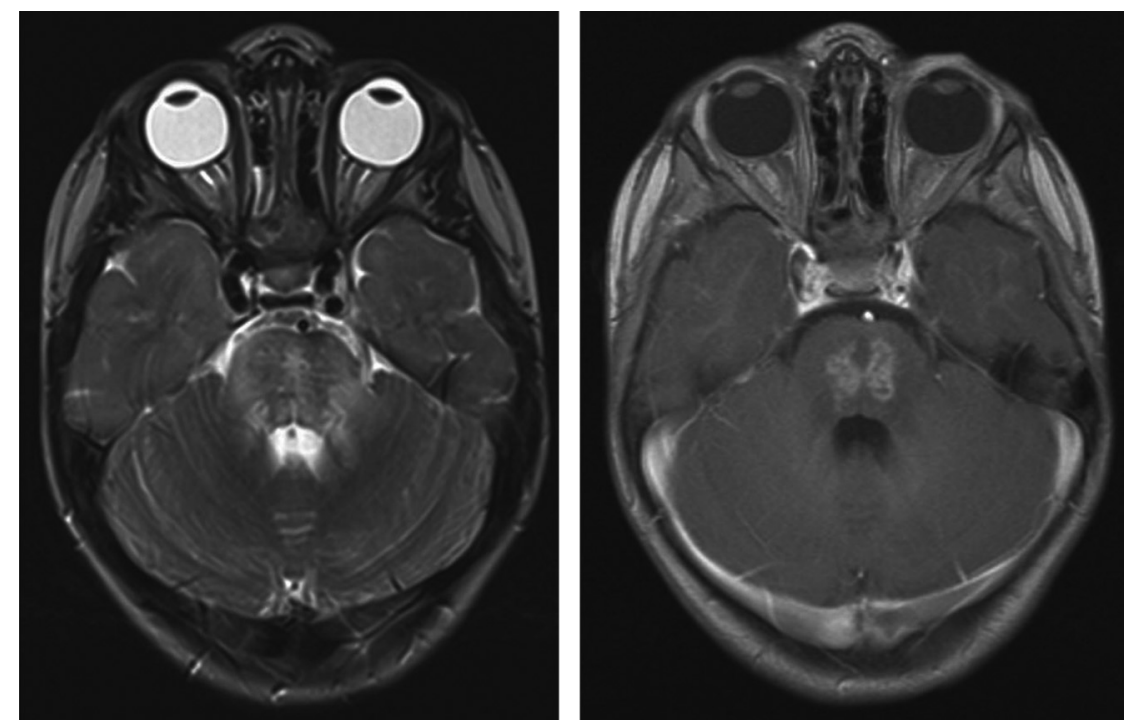

FIG 1. Axial T2-weighted (left) and axial postgadolinium T1-weighted (right) images through the posterior fossa of patient 2, acquired 3.6 months after completion of PT, reveal prominent T2 prolongation and heterogeneous enhancement within the pons. This portion of the pons was in the high-dose volume of irradiation. 

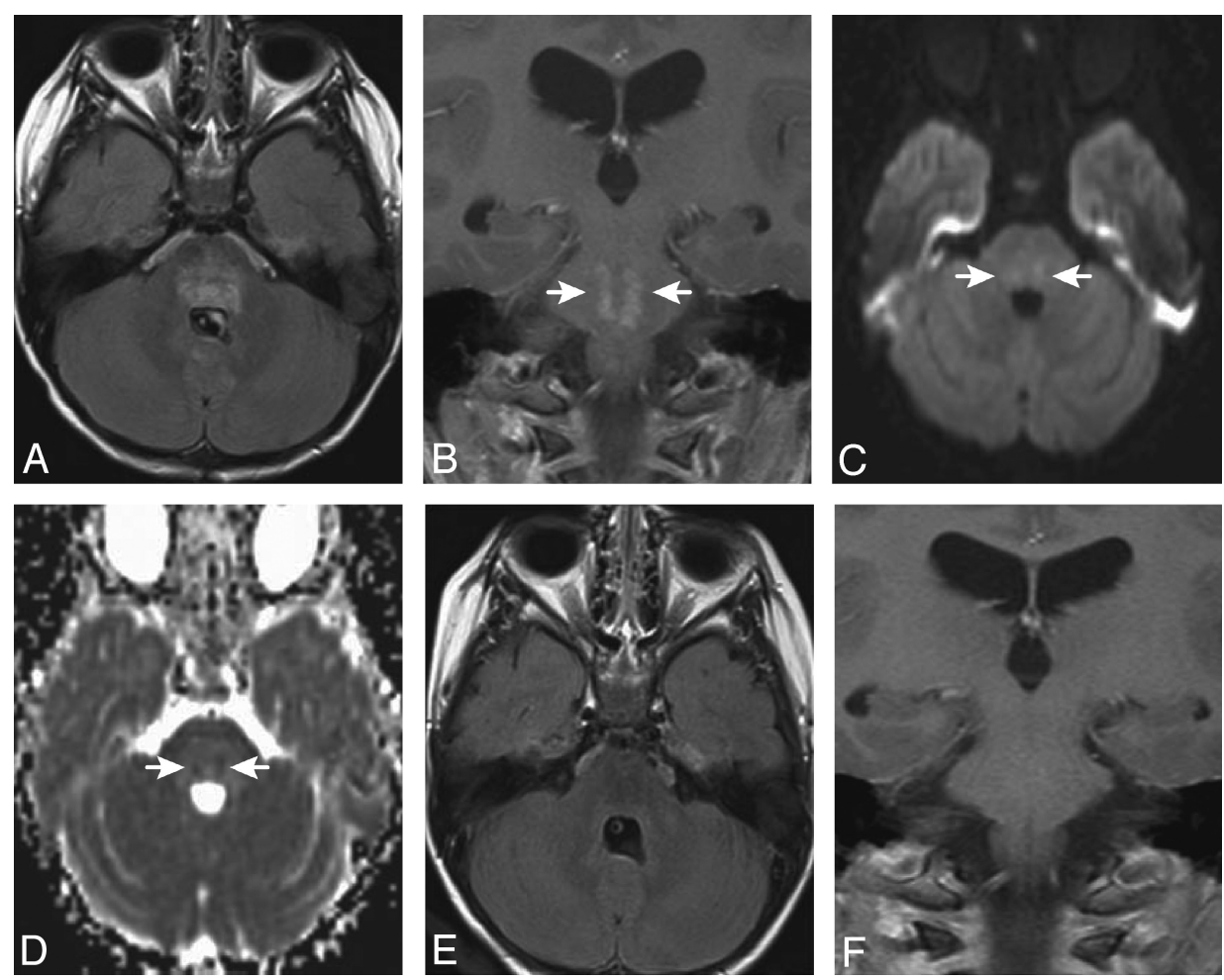

FIG 2. Axial FLAIR $(A)$ and postgadolinium coronal $\Pi$-weighted $(B)$ images for patient 6 , obtained 4.2 months after completion of PT, demonstrate patchy hyperintensity (FLAIR) and focal irregular enhancement (arrows in $B$ ) within the pons. Diffusion-weighted $(C)$ and apparent diffusion coefficient map $(D)$ images reveal small areas of restricted diffusion in the areas of abnormal signal and enhancement. This portion of the pons was in the high-dose volume of irradiation. The abnormalities improved on an MR imaging study obtained 1.5 months later and were no longer visible 4 months after they first appeared $(E$ and $F$ ).
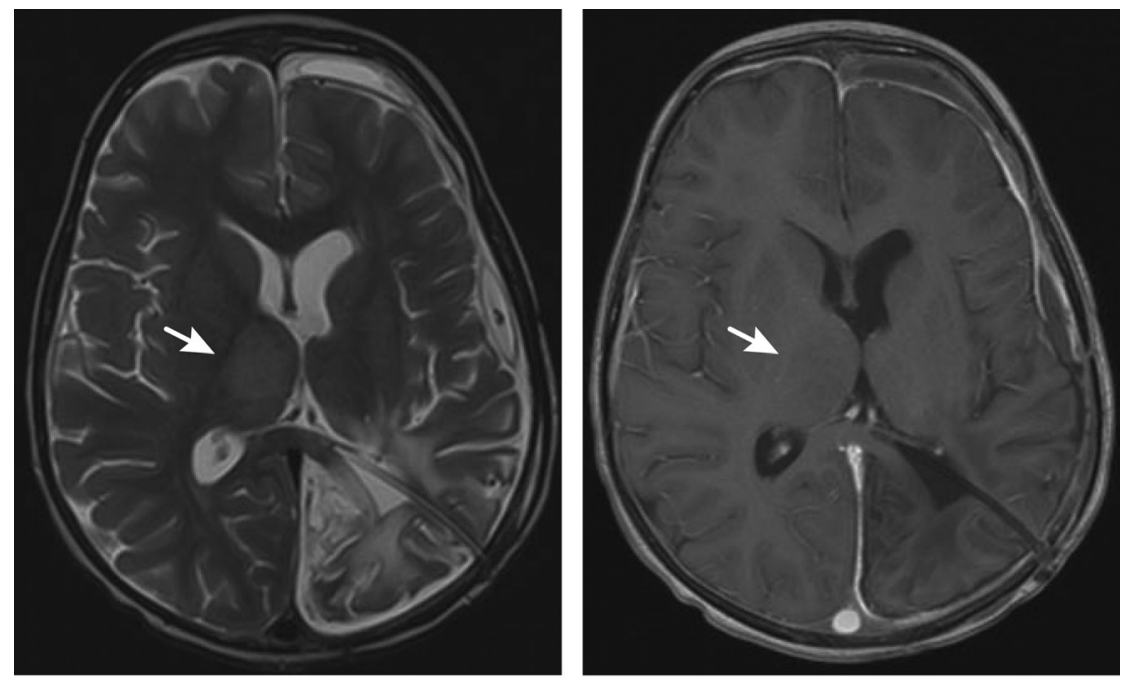

FIG 3. Axial T2 (left) and axial postgadolinium T1-weighted (right) images through the level of the thalami for patient 4 demonstrate enlargement of the right thalamus with hyperintensity on the T2-weighted image without enhancement (arrows).

or complete resolution of the findings. A patient with synchronous extracranial AT/RT with MR imaging changes noted at 3 months posttreatment died of progressive extracranial tumor before a subsequent follow-up study could be performed. In 1 patient, the posterior fossa abnormalities increased on a follow-up study performed 2.1 months later. This patient developed progressive symptoms and imaging evidence of necrosis. The symptoms and imaging changes subsequently improved with hyperbaric oxygen therapy though some imaging changes persisted. A follow-up MR imaging study for the patient with the right thalamic lesion, acquired at an outside institution 1 month after the abnormality was first detected, showed an increase in the size of the abnormal signal before the finding gradually decreased in prominence on later studies. Most patients with imaging changes in our series had posterior fossa tumors and clinical evidence of tumor and surgery-related brain stem manipulation with postoperative neurologic sequelae. Only 1 patient in the current series had more than 1 surgical procedure. Four of the children (patients 2, 4,6 , and 8 ) had subtle and transient neurologic signs related to their post-PT lesions, including unilateral weakness $(n=3)$ or increased ataxia $(n=1)$.

The areas of greatest imaging change were contoured on coregistered MR images and PT dose distributions for each patient. For 6 of the 8 patients, the areas of abnormal signal and enhancement approximately corresponded to areas of high dose (Fig 4). Among the 9 patients who did not show imaging changes after PT, 5 had supratentorial tumors $($ PNET $=4$, ependymoma $=1)$ and 4 had posterior fossa tumors (ependymoma $=$ $3, \mathrm{AT} / \mathrm{RT}=1$ ) in contrast to the 8 patients who developed radiation-associated changes, of which most (6/8) were posterior fossa tumors. The median age at diagnosis for the children who did not have changes after PT was 1.9 years, and the median age at the start of PT was 2.4 years, similar to the median age of diagnosis and start of PT of 1.8 and 2.5 years, respectively, in the patients with PT-related changes. One patient received only 50.4 CGE of therapy rather than the complete 54 CGE and died of progressive disease 8 months after completion of PT. Another patient, without evidence of radiation effect, died of progressive disease approximately 9.5 months after receiving 54 CGE. 
Table 2: MRI findings observed in 8 patients after proton therapy

\begin{tabular}{|c|c|c|c|c|}
\hline & $\begin{array}{l}\text { Time from } \\
\text { Completion of Proton } \\
\text { Therapy to First MRI } \\
\text { with Changes (mo) }\end{array}$ & $\begin{array}{l}\text { Time from Appearance of } \\
\text { Changes to Start of } \\
\text { Resolution of Changes (mo) }\end{array}$ & $\begin{array}{l}\text { Location of Abnormal } \\
\text { Signal/Enhancement }\end{array}$ & $\begin{array}{l}\text { Physical } \\
\text { Examination } \\
\text { Findings }\end{array}$ \\
\hline \multicolumn{5}{|l|}{ Patient } \\
\hline 1 & 5.3 & 2.2 & $\begin{array}{l}\text { Brain stem, cerebellum, upper cervical } \\
\text { spinal cord }\end{array}$ & None \\
\hline 2 & 3.6 & 2.3 & Brain stem & Unilateral weakness \\
\hline 3 & 5.6 & 3 & Cerebellum & None \\
\hline 4 & 3.4 & 3.1 & Right thalamus (no enhancement) & Unilateral weakness \\
\hline 5 & 6.3 & 2.2 & Brain stem & None \\
\hline 6 & 4.2 & 1.5 & Brain stem and cerebellum & Unilateral weakness \\
\hline 7 & 3 & $\begin{array}{l}\text { Patient died of disease before } \\
\text { follow-up }\end{array}$ & Brain stem & None \\
\hline 8 & 3.2 & 3.5 & Brain stem & Ataxia \\
\hline Median & 3.9 & 2.3 (of 7 with follow-up MRI) & & \\
\hline Mean & 4.3 & 2.5 (of 7 with follow-up MRI) & & \\
\hline
\end{tabular}

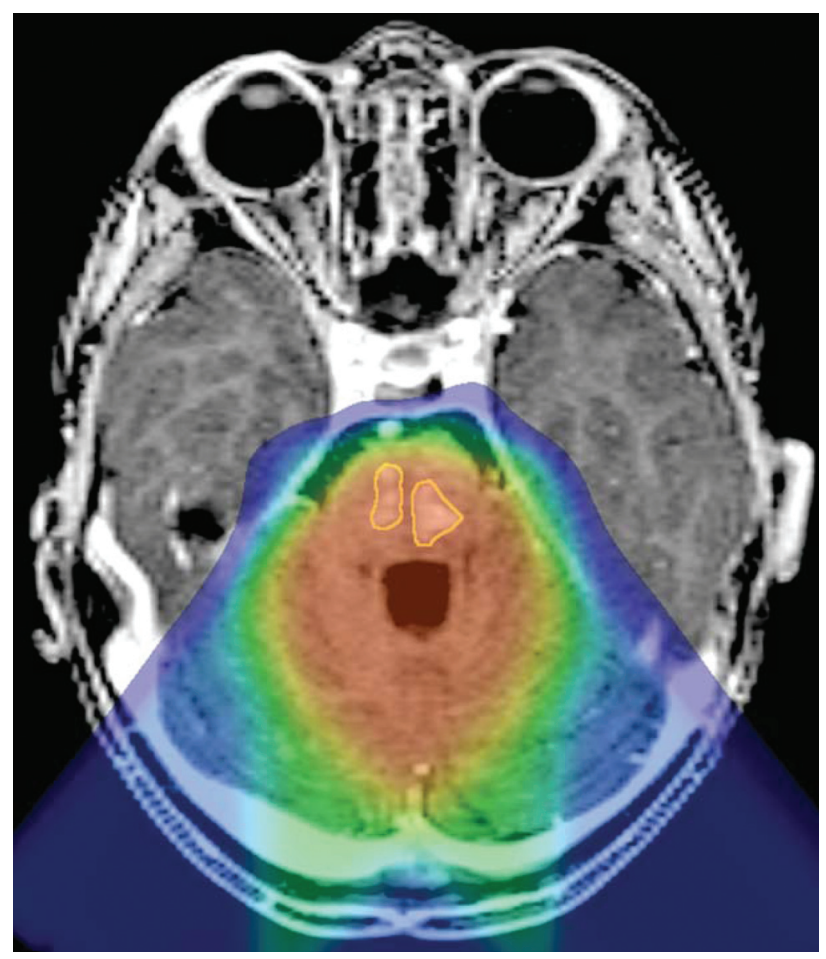

FIG 4. Reformatted axial postgadolinium T1-weighted magnetization-prepared rapid acquisition of gradient echo image through the posterior fossa of patient 8 , coregistered to the proton therapy treatment plan, demonstrates 2 areas of enhancement within the pons (outlined in yellow) located within the high-dose volume of irradiation (orange wash).

\section{DISCUSSION}

Eight of 17 young children with localized brain tumors treated with PT as part of a prospective protocol developed mostly transient imaging changes at a median time of 3.9 months and a mean time of 4.2 months after the completion of PT. The median time to resolution or the beginning of resolution of the imaging changes was 2.3 months.

Radiation therapy-related changes in the pediatric brain on MR imaging are similar to those seen in adults. Acute radiation-induced effects are those observed up to 6 weeks after treatment. ${ }^{3}$ These are often characterized by T1 and T2 pro- longation associated with edema without enhancement. ${ }^{3}$ Early delayed radiation-induced effects are those observed 3 weeks to a few months after therapy. ${ }^{3}$ These are characterized by focal or multiple T1 hypointense and T2 hyperintense white matter lesions, with or without enhancement, and may represent demyelination or edema. ${ }^{3}$ Late delayed radiation-induced effects are observed several months to years after treatment and are characterized by $\mathrm{T} 1$ and $\mathrm{T} 2$ prolongation, enhancement, necrosis, and mass effect. ${ }^{3}$ The time course for presentation and regression of the lesions in our 8 patients is compatible with an early delayed radiation-therapy effect, the contributions of pre- and postirradiation chemotherapy notwithstanding.

The imaging appearance of PT-related lesions in this series-mostly transient, T2 hyperintense enhancing lesions with associated $\mathrm{T} 1$ hypointensity and, in most cases, postgadolinium enhancement-is similar to that described in children treated with photon radiation therapy. ${ }^{4-7}$ However, median and mean ages at diagnosis and treatment of the patients in our series, 1.8 and 2.4 years respectively, were much younger than the median ages of 7.1 years and 8.1 years at presentation in 2 of those series $^{6,7}$ and mean age of 5.4 years in 1 of those studies. ${ }^{5}$ Median and mean times to lesion development in our series of children post-PT are shorter than the time to appearance of photon radiation therapy-related lesions, which have been reported to arise a median of 6-10 months after the start or completion of radiation therapy. ${ }^{4-7}$ The lesions in our series also tended to regress sooner (1.5-3.5 months) than the time interval of 2-26 months described for patients treated with photons. ${ }^{4-7}$ The differences in the time course may be related to the use of PT, the induction or maintenance chemotherapy regimen, the age of the patients included in our series, ${ }^{8,9}$ or other factors coincident with the performance of the protocol, including surgical sequelae. ${ }^{10-12}$

The signal changes and enhancement demonstrated in our series of children resemble the imaging findings of tumor pseudoprogression. Pseudoprogression is an increase in MR imaging findings of edema and enhancement in an area of tumor, suggestive of tumor progression, after a patient has received combination radiation and chemotherapy. The findings characteristically diminish or resolve on follow-up studies, with the patient not receiving additional ther- 
apy. ${ }^{13,14}$ The findings of pseudoprogression typically occur within 3 months after completion of treatment, though the imaging changes may first appear up to 6 months after therapy ends. ${ }^{14}$ Although the MR imaging changes for all except 1 of the children in our series fit within the timeframe for pseudoprogression, the abnormalities in our patients were not within the areas from which tumor was resected and were not located within structures to which the treated neoplasms characteristically extend. The findings in our patient series, therefore, are not in keeping with pseudoprogression.

Although several prior studies have described imaging changes after PT in the brains of adult patients treated for skull base tumors, ${ }^{12,15,16}$ description of PT-related imaging findings in the brain in pediatric patients is limited to a report of a 14-year-old boy treated with $54 \mathrm{~Gy}$ without chemotherapy for residual tectal oligodendroglioma along the left thalamus following resection of recurrent disease, who subsequently developed left thalamic enhancement and mild associated mass effect 6 months after PT, which resolved 9 months later following a 1-month course of corticosteroids. ${ }^{17}$ Ours is the first series to systematically document PT-related imaging changes in young children with brain tumors following chemotherapy. We observed that the onset of imaging changes and regression was earlier after the completion of PT than that observed in previous reports of patients treated with photon radiation therapy. We also noted that the imaging changes usually had minimal clinical significance. Beyond the inherent susceptibility of the pediatric brain to therapy-induced neurotoxicity, the combined effects of gross-total resection, pre- and postirradiation chemotherapy, and the inherent physical and radiobiologic differences of protons compared with photons should be considered as potential causes of the MR imaging findings. Further study is necessary to clarify the pathophysiology of PT-related imaging changes.

\section{ACKNOWLEDGMENTS}

We thank Mary Baltimore for her assistance in the preparation of this manuscript.

Disclosures: Jonathan Gray- UNRELATED: Other: Philips, Comments: focus group honorarium. Daniel Indelicato_UNRELATED: Consultancy: Arthur D. Little, Comments: consultant on proton therapy survey $2012(<\$ 500)$.

\section{REFERENCES}

1. Merchant TE, Li C, Xiong X, et al. Conformal radiotherapy after surgery for paediatric ependymoma: a prospective study. Lancet Oncol 2009; 10:258-66

2. Yock TI, Tarbell NJ. Technology insight: proton beam radiotherapy for treatment in pediatric brain tumors. Nat Clin Pract Oncol 2004;1:97-103

3. Ball WS, Prenger EC, Ballard ET. Neurotoxicity of radio/chemotherapy in children: pathologic and MR correlation. AJNR Am J Neuroradiol 1992;13:761-76

4. Fouladi M, Chintagumpala M, Laningham FH, et al. White matter lesions detected by magnetic resonance imaging after radiotherapy and high-dose chemotherapy in children with medulloblastoma or primitive neuroectodermal tumor. J Clin Oncol 2004;22:4551-60

5. Helton KJ, Edwards M, Steen RG, et al. Neuroimaging-detected late transient treatment-induced lesions in pediatric patients with brain tumors. J Neurosurg 2005;102:179-86

6. Spreafico F, Gandola L, Marchiano A, et al. Brain magnetic resonance imaging after high-dose chemotherapy and radiotherapy for childhood brain tumors. Int $J$ Radiat Oncol Biol Phys 2008;70:1011-19

7. Muscal JA, Jones JY, Paulino AC, et al. Changes mimicking new leptomeningeal disease after intensity-modulated radiotherapy for medulloblastoma. Int J Radiat Oncol Biol Phys 2009;73:214-21

8. Lafay-Cousin L, Strother D. Current treatment approaches for infants with malignant central nervous system tumors. Oncologist 2009; 14:433-44

9. Duffner PK, Horowitz ME, Krischer JP, et al. The treatment of malignant brain tumors in infants and very young children: an update of the Pediatric Oncology Group experience. Neuro Oncol 1999;1:152-61

10. Kellie SJ, Chaku J, Lockwood LR, et al. Late magnetic resonance imaging features of leukoencephalopathy in children with central nervous system tumors following high-dose methotrexate and neuraxis radiation therapy. Eur J Cancer 2005;41:1588-96

11. Packer RJ, Grossman RI, Rorke LB, et al. Brain stem necrosis after preradiation high-dose methotrexate. Childs Nerv Syst $1985 ; 1: 355-58$

12. Debus J, Hug EB, Liebsch NJ, et al. Brainstem tolerance to conformal radiotherapy of skull base tumors. Int J Radiat Oncol Biol Phys 1997;39:967-75

13. Brandsma D, Stalpers L, Taal W, et al. Clinical features, mechanisms, and management of pseudoprogression in malignant gliomas. Lancet Oncol 2008;9:453-61

14. Hygino da Cruz LC Jr, Rodriguez I, Domingues RC, et al. Pseudoprogression and pseudoresponse: imaging challenges in the assessment of posttreatment glioma. AJNR Am J Neuroradiol 2011;32:1978-85

15. Santoni R, Liebsch N, Finkelstein DM, et al. Temporal lobe (TL) damage following surgery and high-dose photon and proton irradiation in 96 patients affected by chordomas and chondrosarcomas of the base of the skull. Int J Radiat Oncol Biol Phys 1998;41:59-68

16. Miyawaki D, Murakami M, Demizu Y, et al. Brain injury after proton therapy or carbon ion therapy for head-and-neck cancer and skull base tumors. Int J Radiat Oncol Biol Phys 2009;75:378-84

17. Meyzer C, Dhermain F, Ducreux D, et al. A case report of pseudoprogression followed by complete remission after proton-beam irradiation for a low-grade glioma in a teenager: the value of dynamic contrast-enhanced MRI. Radiat Oncol 2010;5:9 